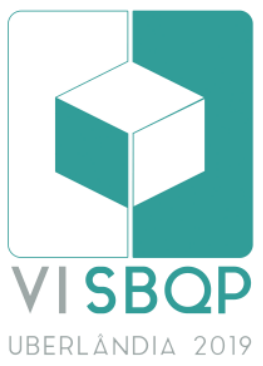

\title{
A IMPORTÂNCIA DA GESTÃO E GERENCIAMENTO EM EMPREENDIMENTOS DE RETROFIT
}

\author{
SILVA, Eduarda \\ Universidade Federal de Minas Gerais, e-mail: eduardasantana12@hotmail.com
}

\begin{abstract}
RESUMO
O esvaziamento e a grande quantidade de edificações desabitadas ou subutilizadas evidenciam a clara necessidade de requalificar as edificações dos grandes centros urbanos brasileiros. O retrofit, responsável por adaptar e reabilitar as edificações, se mostra como muito eficaz nesse contexto, ainda mais se considerarmos a infraestrutura disponível nestes locais. Infelizmente, existem poucas informações específicas sobre a gestão e o gerenciamento desse tipo de empreendimento. Este trabalho tem por objetivo contribuir para o avanço do conhecimento na área de retrofit, demonstrando a necessidade de realizar a gestão e o gerenciamento de empreendimentos desse tipo, enfatizando a importância de compreender a obra de retrofit de forma sistêmica, a fim de antever, através destes sistemas, os riscos envolvidos em um retrofit arquitetônico. O trabalho foi realizado por meio de consulta à literatura disponível, levantamento de conceitos sobre o retrofit, gerenciamento, gestão de obras e gestão de riscos. O que fica evidenciado com esta pesquisa é a ligação direta entre a realização da gestão e gerenciamento de obras de retrofit e o resultado do empreendimento.
\end{abstract}

Palavras-chave: retrofit, Gestão e Gerenciamento, Gestão de Riscos em retrofit.

\begin{abstract}
The Population depletion and the big quantity of uninhabited or underutilized buildings, show the need to requalify the big Brazilians urban centers buildings. The retrofit, responsible for adapting the buildings, is presented as very relevant in this context, even more if we consider the available infrastructure of these places. Unfortunately, there is little specific information about the management of this kind of project. This paper aims to contribute to advancing retrofit knowing, demonstrating the need to realize the management of this kind of project, underlying the importance to know the retrofit project in a systemic way, in order to foresee, using these systems, the risks associated with an architectural retrofit. This paper was made through the consultation of available scientific literature, identification of retrofit and management of projects and risks concepts. What is evident from this research is the direct link between the management of retrofit project realization and the project result
\end{abstract}

Keywords: retrofit, Management, Management of risks in retrofit.

\section{INTRODUÇÃO}

A expansão das grandes metrópoles brasileiras fez com que, nas últimas décadas, ocorresse um processo de esvaziamento populacional dos centros urbanos, propiciando o surgimento de espaços ociosos. Grande parte da população acaba migrando para novas regiões em busca de edifícios mais modernos, contribuindo para a degradação do centro das cidades.

Uma das formas de contribuir para reverter este processo são as obras de retrofit, responsáveis por adaptar as edificações e torná-las compatíveis com as necessidades e princípios da atualidade, valorizando também a região em

SILVA, E. A Importância da Gestão e Gerenciamento em Empreendimento de Retrofit. In: SIMPÓSIO BRASILEIRO DE QUALIDADE DO PROJETO NO AMBIENTE CONSTRUÍDO, 6., 2019, Uberlândia. Anais... Uberlândia: PPGAU/FAUeD/UFU, 2019. p. 20-28. DOI https://doi.org/10.14393/sbqp19003. 
que ela está localizada.

No Brasil, principalmente devido ao aumento da preocupação com as áreas tombadas e ao desenvolvimento dos centros urbanos, o retrofit aparece como tendência de mercado Vale (2006. p.96).

Nas principais cidades o que acaba acontecendo é uma disputa por cada metro quadrado vazio, cada vez mais escassos, enquanto edificações com ótimas localizações e infraestrutura vão ficando sem uso, subutilizadas, obsoletas e comercialmente inviáveis. É neste contexto que percebemos a reabilitação de edifícios como uma oportunidade para a construção civil.

Este estudo tem como objetivo contribuir para o avanço do conhecimento na área de retrofit, demonstrando a necessidade de realizar a gestão e o gerenciamento de empreendimentos de retrofit, enfatizando a necessidade de compreender estas obras de forma sistêmica, a fim de antever, através de um sistema de gestão e gerenciamento, os riscos envolvidos, visto que é um tipo de empreendimento onde os riscos/imprevistos tem uma taxa elevada, se comparada a empreendimentos de engenharia comum.

Para isso, conhecer mais sobre o processo de retrofit e como se dá seu gerenciamento é de suma importância para o empreendimento, visto que possibilita exercer controle sobre os riscos envolvidos durante todas as fases do projeto.

Dentro deste contexto, acredita-se na importância de realizar um diagnóstico das condições físicas do edifício a ser reabilitado, antes da concepção do projeto, para que o retrofit atinja o objetivo desejado

\section{METODOLOGIA}

Para realização do levantamento de dados e análises necessárias para elaboração do presente trabalho, o tema foi dividido em subitens para possibilitar um melhor entendimento, sendo esses: Reabilitação de edifícios, Gerenciamento e Gestão de riscos. Para cada subitem, foram realizados levantamentos bibliográficos, incluindo livros, teses, dissertações e monografias, levando em consideração as referências que possibilitassem analisar as principais particularidades de empreendimento de retrofit, entraves e benefícios envolvidos com o processo.

\section{FUNDAMENTAÇÃO TEÓRICA}

\subsection{Reabilitação de edifícios}

A reabilitação de edifícios engloba vários tipos de intervenções possíveis a um edifício. Com o objetivo de levantar e propor soluções, problemas decorrentes da sua utilização e falta de utilização/degradação, de forma a possibilitar o aumento da vida útil da edificação por meio da recuperação e modernização dos sistemas das instalações.

Com base em Jesus (2008), propõe-se a seguinte classificação das formas de reabilitação de edifícios: restauração, relativo à preservação do Patrimônio Histórico edificado, que exigem extremo cuidado em conservar aspectos históricos da edificação; manutenção e reparo, referente a conservação da edificação, que pode ser entendido como os cuidados necessários durante a vida de uma edificação e; reforma e retrofit, voltados para o aumento da vida 
útil do edifício sem compromisso em recuperar características originais e promovendo a utilização de novas tecnologias.

De acordo com Morettini (2012), independente de qual intervenção será utilizada para reabilitação do edifício é importante entender as particularidades de cada processo para o sucesso do empreendimento. Neste artigo vamos tratar de reabilitações pelo processo de retrofit.

\subsection{Retrofit}

De acordo com Vale (2006), a expressão retrofit significa, em sua origem: ajuste, adaptação. Inicialmente era utilizada para designar a atualização tecnológica pela qual as aeronaves passavam quando adquiriam equipamentos mais modernos. Com o passar dos anos o termo foi incorporado na construção civil.

Com o significado de "colocar o antigo em boa forma", o termo vem sendo utilizado para práticas de revitalizar antigos edifícios, incorporando novas tecnologias, materiais, e sempre que possível acessibilidade, a fim de, adaptálos às novas demandas de uso além de, sempre que possível atender às normas técnicas.

Sendo assim, o retrofit se apresenta como uma alternativa que promova bemestar, segurança, utilidade, acessibilidade e, que seja viável economicamente. Esta proposta é uma forma inteligente e amplamente sustentável de recuperar e adaptar as edificações, permitindo novamente sua utilização.

De acordo com Vale (2006) o retrofit pode ser classificado em 4 graus de abrangência: rápido: que envolve recuperar instalações e revestimentos internos; médio: que abrange intervenções em fachadas; profundo: que contempla as atividades anteriores e agrega mudanças de layout e telhado; e por fim, o retrofit excepcional que ocorre em edificações históricas, como exemplos desse tipo de retrofit, é possível citar os trabalhos realizados pelo IPHAM e Docomomo que tem a preocupação de incentivar os processos do retrofit em edificações tombadas ou com importância histórica.

Conclui-se então que o retrofit arquitetônico utiliza de estratégias para manter a edificação atual às demandas de uso, escapando da decadência, mantendo sua estrutura original, mas apostando em atualizações tecnológicas e de materiais, em novos usos para seus espaços, evitando a obsolescência e, acompanhando o desenvolvimento tecnológico da construção civil.

\subsection{Gerenciamento e gestão de projeto}

Para melhor entendimento, devemos conceituar o termo projeto:

Projeto é um esforço temporário empreendido para criar um produto, serviço ou resultado exclusivo. A natureza temporária dos projetos indica que eles têm um início e um término definidos. O término é alcançado quando os objetivos do projeto são atingidos ou quando o projeto é encerrado porque os seus objetivos não serão ou não podem ser alcançados, ou quando a necessidade do projeto deixar de existir. Guia PMBOK (2012, p.3).

O resultado dos projetos é único. Afinal, eles precisam atingir um objetivo claro, dentro de um prazo determinado, contando com um conjunto limitado de recursos (humanos, financeiros e materiais) que precisam ser otimizados para alcançar as metas estipuladas, sem exceder as expectativas iniciais de seus 
patrocinadores.

O gerenciamento de projetos pode ser entendido como um sistema de práticas padronizadas que buscam produzir resultados pelos variados sistemas de gestão.

Existem várias áreas em que um sistema de gestão é necessário durante uma obra, por exemplo: gestão de recursos e materiais, gestão da qualidade, gestão ambiental, gestão de relacionamento com o cliente, dentre outros. Sendo o propósito final do sistema de gestão é assegurar às organizações o gerenciamento através de estratégias organizacionais.

\subsection{Obras de retrofit e gerenciamento}

Cada obra de Engenharia Civil possui suas especificidades e peculiaridades. Por isso, cada obra vai necessitar do seu próprio sistema de gerenciamento. Mas se considerarmos que mesmo em obras novas, onde existe uma grande padronização de serviços, é necessário um cuidado com o gerenciamento, quando falamos de obras de retrofit, este cuidado deve ser ainda maior, por atuar sobre um projeto existente, sem a mesma padronização.

De acordo com Lima (2016), é mais complexo e difícil de alcançar o nível de exatidão desejado em obras de retrofit, devido à dificuldade de planejar, prever tempo e custo e executar.

Considerando a complexibilidade das obras de retrofit, é recomendado que o projetista tenha experiência com esse tipo de empreendimento, visto que ele tem demandas importantes a serem consideradas, por exemplo: sustentabilidade, relevância arquitetônica e desempenho da edificação.

De acordo com Moraes e Quelhas (2012), a fase de planejamento do retrofit pode ser dividida em: Análise mercadológica e financeira, Definição do conceito do projeto, Estudo da Legislação, Diretrizes para reaproveitamento de materiais e sistemas, Diagnóstico, Propostas de implementação do projeto e Comercialização.

Dentre todas as etapas apresentadas, a etapa de diagnóstico é, sem dúvidas, a mais importante em um empreendimento de retrofit. Esta etapa visa realizar - levantamento e ainda obter informações sobre o histórico da construção, estando intimamente ligada ao sucesso ou fracasso do projeto.

É usual existir dificuldades em se obter informações, devido à falta de material disponível. Mas é preciso deixar claro que, não se deve desprezar informações importantes que possivelmente vão gerar impactos no andamento da obra. Quando encontrados, os documentos devem ser colocados a prova através de medição e análises in loco, visto que, devido à grande lacuna de tempo, a possibilidade de incompatibilidade é grande.

Todo esse cuidado com obras de retrofit é necessário, pois compreende avaliar um projeto existente, que pode ter sofrido alterações posteriores, que não estão documentados, visando aumentar a sua longevidade, o que pode gerar supressas durante a obra, por isso é necessário realizar uma cuidadosa avaliação física evitando comprometer a qualidade dos projetos de intervenção com informações falsas.

Após a etapa de levantamento e síntese de todas as informações recolhidas, obtemos um diagnóstico das condições do edifício. Por meio disso, é possível tomar as melhores decisões para realizar o processo de retrofit.

O conjunto de todas essas informações recolhidas e organizadas 
proporcionará à equipe de projeto as informações necessárias para realizar os alinhamentos entre as potencialidades e as necessidades daquele projeto.

Appleton (2003), destaca a importância de se conhecer a edificação completamente, afim de definir um programa base para essa intervenção, ou seja, partir da edificação que se tem, para a que se deseja ter.

Zarur (2017), afirma que a partir do momento em que as questões legais estão solucionadas e o projeto conceitual de arquitetura foi elaborado, passa-se para a etapa de desenvolvimento dos projetos complementares, que serão desenvolvidos e compatibilizados.

É importante ressaltar que, qualquer que seja o projeto, ele deve ser pensando considerando o levantamento feito incialmente e suas atualizações. Jamais, em uma obra de retrofit deve-se propor um elemento sem conversar com os demais componentes da obra.

Deve-se salientar que uma etapa crucial para uma obra de retrofit é o planejamento de canteiro. É indicado que se realize um estudo de implantação de canteiro, juntamente com o desenvolvimento do projeto, evitando dificuldades para alocar material e trabal hadores na obra.

Após a aprovação de todos os projetos e a comercialização de parte do empreendimento, inicia-se a etapa executiva da obra, na qual toda a parte anterior é colocada em prática. Por melhor que seja a qualidade dos trabalhos realizados nas etapas de diagnóstico, sempre é possível encontrar problemas na etapa de execução das obras, em função de interferências não previstas anteriormente. Devido a essa situação, se destaca a importância do gerenciamento de risco e a presença de um arquiteto ou engenheiro responsável para realizar o acompanhamento de forma regular e metódica, evitando erros de execução e atrasos na obra. O estudo de novas alternativas também deve ser feito com o decorrer do avanço da obra. Considerando que a possibilidade de imprevistos é real.

Em resumo, uma obra de retrofit possui riscos mais elevados que obras convencionais, por lidar com uma edificação existente. Esta situação vai exigir dos profissionais muita perícia, controle financeiro e de prazos, para que as possíveis surpresas ao longo do trabalho não prejudiquem o projeto.

\subsection{Gestão de riscos}

Atualmente a ISO 31000:2018 define risco como, "O efeito da incerteza nos objetivos". Um efeito é um desvio em relação ao esperado. Pode ser positivo, negativo ou ambos, e pode abordar, criar ou resultar em oportunidades e ameaças.

Gestão de riscos é um conjunto de atividades coordenadas que visa identificar, analisar e planejar ações a fim de evitar situações indesejadas ou potencializar oportunidades. ISO $31000(2018$, p.2).

O propósito da ISO 31000:2018 é a criação e proteção de valor. Melhorando o desempenho, encorajando a inovação e apoiando o alcance dos objetivos. Para isso ela fornece orientações sobre as características de uma gestão de riscos eficiente e eficaz.

A ISO 31000:2018 não constitui uma norma para a implementação de um sistema de gestão, mas é um guia para a aplicação da gestão do risco. Sendo assim, a principal diferença entre esta norma e outros guias e documentos 
relativos à gestão do risco, consiste na introdução de uma estrutura do processo de gestão do risco.

Ao longo das últimas décadas tem sido propostos vários modelos que estruturam as várias fases que compõe um processo de gestão de riscos. Um dos modelos do processo de gestão de riscos mais relevante atualmente para o mercado brasileiro é o PMBOK.

Segundo o Guia PMBOK, o gerenciamento dos riscos inclui seis processos, buscando sempre antecipar possíveis respostas para pontos de vulnerabilidade do projeto. Seu objetivo é maximizar a exposição aos eventos positivos e minimizar a exposição aos eventos negativos.

As empresas, habitualmente, estão dispostas a aceitar vários níveis de riscos em seus empreendimentos, isso vai depender da sua atitude em relação aos riscos, que pode ser influenciada por inúmeros fatores. O que não deve ocorrer em um projeto ou obra é a falta de gestão de riscos, podendo resultar no aumento de custos e prazos estimados e, em casos mais graves impedir sua conclusão.

Comumente, os objetivos de um projeto são determinados com base no custo da obra, no tempo de execução, e na qualidade final esperada. A partir de então, as incertezas vinculadas a cada um desses fatores devem ser estudadas e analisadas. Ressaltando que, não apenas os riscos negativos devem ser investigados, mas também os positivos que podem vir a se tornar uma potencialidade do empreendimento no futuro.

Zarur (2017), salienta que o processo de gerenciamento de riscos, tende a ser frágil, por se tratar de atividades que envolvam pessoas e, qualquer processo que envolva pessoas tende a ser sensível e de difícil resolução.

Atualmente o mercado da indústria civil conta com diversos programas e metodologias para simplificar o processo da gestão de riscos, tornando-o mais seguro e eficaz, sendo importante para o sucesso, que o plano de gerenciamento de riscos considere os todos os planos auxiliares e as linhas de base aprovadas, sendo parte integrante do plano de gerenciamento do projeto.

\subsection{Gestão de riscos em retrofit}

Apesar de existir muita informação e inúmeras publicações acerca do risco e da gestão do risco na construção, o tema da reabilitação tem ainda pouca expressão em termos da informação disponível. Correia (2015, p.32).

Podemos considerar que riscos são todos os acontecimentos não previstos. É evidente que os riscos negativos são os mais importantes de serem mensurados, por terem potencial para causar atrasos, aumento de gastos e inviabilizar uma obra. Mas o conhecimento das potencialidades do projeto é um ponto fundamental do seu planejamento estratégico e sua diferenciação no mercado atual.

Videira (2006) apud Correia (2015), identifica os principais riscos em empreendimentos de reabilitação de edificações, que podem ser classificados como: intrínsecos a edificação, como limitações técnicas, localização e falta de documentação e; intrínsecos a equipe de projeto e execução, como falta de conhecimento acerca da edificação, falta de clareza para estipulação de prazos e custos, trabalhar com uma equipe sem 
experiência com projetos similares, não realizar uma boa coordenação de projetos e a inexistência da pormenorização da solução pretendida.

Em projetos de reabilitação, existe uma grande falta de padronização, o que aumenta substancialmente os riscos envolvidos. A insegurança, gerada pelos imprevistos, é algo muito relevante para os investidores desse tipo de empreendimento. Tais riscos evidenciam a importância de se obter conhecimento de qualidade sobre o empreendimento.

De acordo com Correia (2015) O grau de incerteza de um projeto está ligado diretamente ao conhecimento que se tem do mesmo. Sendo que os riscos diminuem, a medida que a qualidade e o número de informações aumenta. Isso reflete principalmente nas etapas preliminares, onde as decisões mais importantes são tomadas e irão impactar no resultado.

A gestão de riscos é um fator fundamental nos projetos de reabilitação, por se tratar de algo pré-existente e, muitas vezes, desconhecido. Estar atento aos riscos apresentados e aos processos de gestão apresentado pelo PMBOK é uma das formas de realizar uma obra de retrofit de maneira segura, evitando problemas como: baixa qualidade no produto acabado, aumento nos custos, atrasos e demais surpresas indesejadas. Portanto, fica claro que o sucesso de um empreendimento, seja ele retrofit ou não, tem uma ligação direta com uma gestão de riscos eficiente.

\subsection{PDRI auxiliando na gestão de riscos}

O PDRI (Project Definition Rating Index) é uma ferramenta utilizada para a análise do nível de detalhamento e maturidade de um projeto.

O PDRI avalia o nível de maturidade de projetos através de um índice que indica qual é a possibilidade de manutenção dos interesses e objetivos iniciais dos empreendimentos, em função dos esforços envolvidos no detalhamento do projeto. Marques, Starling e Andery (2015, p.3).

Através dele é possível avaliar os riscos integrantes ao projeto analisado. A ferramenta em questão é um questionário auto avaliativo, que aborda 64 pontos classificados como importantes para indicar se um projeto foi planejado detalhadamente.

Segundo Marques, Starling e Andery (2015, p.6), o questionário do PDRI, aborda problemas de ineficiência em obras. Realizando avaliações sobre: concepção de projeto, disposição de recursos orçamentários, qualidade dos projetos e planejamento da construção.

O resultado desta autoavaliação é uma pontuação de nível de definição de projeto, que é usada para medir o nível de vulnerabilidade e os riscos do empreendimento. Usualmente as questões tratadas pelo PDRI, devem ser tratadas na etapa de pré-planejamento do projeto.

O PDRI é feito com base em uma avaliação numérica da definição dos elementos em uma escala de 0 a 5 . Sendo a nota 0 utilizada para um elemento não aplicável ao projeto, 1 representa uma definição completa de escopo, 2 para deficiências menores, 3 apresentam algumas deficiências, 4 para maiores deficiências e, 5 representam uma definição pobre e incompleta.

Com isso, um projeto que obtenha pontuação total igual ou menor que 2, é considerado um projeto com grandes possibilidades de sucesso. Deve-se 
salientar que, quanto menor esse número, mais maturidade o projeto apresenta.

De acordo com Correia (2015), o PDRI é uma ferramenta, eficiente, rápida e simples que pode ser usada ao longo do processo de concepção e planejamento do projeto, visando garantir alinhamento contínuo. É importante destacar que o PDRI tem como base, as boas práticas do mercado, utilizandose de pesos diferentes para classificar seus itens analisados. Isso ocorre de acordo com a influência que cada item pode vir a ter na obra.

Wang (2002) demonstra em seu estudo que realizar um escopo detalhado de um projeto aplicando posteriormente o PDRI amplia significativamente a chance de sucesso do projeto

Utilizar desse recurso para gestão de riscos de empreendimentos apresenta-se como um caminho para obter melhores resultados, principalmente em relação ao tempo e o custo. Diante do resultado do PDRI os responsáveis devem decidir seguir com o projeto ou sanar as deficiências encontradas.

\section{CONSIDERAÇÕES FINAIS}

A Requalificação é um tema atual, que se apresenta como uma solução mais sustentável e viável economicamente, sendo uma possibilidade eficaz de dar uso às edificações desocupadas e em estado de degradação, em locais dotados de infraestrutura e possibilidades de autonomia econômica.

Fica evidente nas análises, que o gerenciamento de projetos de retrofit, influencia positivamente para o sucesso de uma obra, pois permite que a reabilitação seja executada nos prazos e custos definidos, assegurando o nível projetado e em consequência a qualidade da obra

Assim como o gerenciamento, a gestão de riscos se apresenta como vigente e de grande importância nas obras de retrofit. Através da gestão do risco, é possível gerir as incertezas que surgem no empreendimento, operando como um auxiliar na tomada de decisão e facilitando o alcance dos objetivos.

Visto que o grau de incerteza intrínseco a uma obra de retrofit é grande, a implementação da gestão de riscos numa organização ou empreendimento permite gerir e tratar essas incertezas de modo eficaz, providenciando um conhecimento atualizado, correto e compreensivo dos riscos, assegurando que o nível de risco é compatível com os critérios estipulados.

Conclui-se que existe uma ligação direta entre uma gestão de riscos eficaz e o sucesso de um empreendimento de retrofit, quando os riscos, positivos e negativos, são identificados, tem-se mais claramente o potencial da obra. As empresas que utilizam esses métodos, tendem a se tornar mais competitivas frente ao mercado da construção civil.

Deste modo entende-se que o estudo efetuado atingiu seu objetivo, contribuindo para a problemática do conhecimento da importância sobre o gerenciamento e da gestão de retrofit arquitetônico. Para isso foram apresentados parâmetros relevantes sobre gerenciamento e gestão presentes nesse tipo de investimento. Contemplando o PDRI como uma ferramenta auxiliar viável nesse processo. 


\section{REFERÊNCIAS}

ABNT NBR ISO. Norma n 31000, de 2018. Gestão de riscos - Diretrizes. Risk management - Guidelines. Disponível em:

https://www.abntcatalogo.com.br/norma.aspx? ID=392334. Acesso em: 14 ago. 2018

APPLETON, José., Reabilitação de edifícios antigos: patologias e tecnologias de intervenção. Amadora: Orion, 2003. p.146.

CORREIA, Mariana Pinto da Rocha Alves. Contributo para a implementação da gestão do risco em obras de reabilitação de edifícios. 2015. Dissertação (Mestre em engenharia Civil) - Faculdade de Lisboa, Lisboa - Portugal, 2015.

JESUS, Christiano R. M.. Análise de custos para reabilitação de edifícios para habitação. Dissertação de mestrado. São Paulo, 2008

LIMA, E., Estudo da Contribuição das Metodologias do Lean Construction e do Gerenciamento de Projetos do PMI para o Planejamento e Controle da Produção de Obras. Projeto de graduação apresentado a Escola Politécnica/ UFRJ, Rio de Janeiro, 2016.

MARQUES, STARLING E ANDERY., Análise e identificação de riscos e incertezas em empreendimentos de incorporação imobiliária, Departamento de engenharia de materiais e construção - UFMG, 2015.

MORAES, V., QUELHAS, O., O Desenvolvimento da Metodologia e os Processos de um Retrofit Arquitetônico, Revista Eletrônica Sistemas \& Gestão, v. 7, n.3, pp 448-461, 2012.

MORETTINI, Renato. Tecnologias construtivas para reabilitação de edifícios:

Tomada de decisão para uma reabilitação sustentável. Dissertação em engenharia civil. São Paulo, 2012.

PROJECT MANAGEMENT INSTITUTE. Conjunto de conhecimentos em

gerenciamento de projetos (guia PMBOK). Pennsylvania: 3 eds. S. I., 2004. 225p

VALE, Mauricio Soares. Diretrizes para racionalização e atualização das edificações: Segundo o conceito da qualidade e sobre a ótica do retrofit. Rio de Janeiro, 2006

ZARUR, Daniela, Batista. Sistermas de gestão e gerenciamento em obras de Retrofit: aspectos teóricos e práticos. Graduação em Engenharia Civil, Rio de Janeiro, 2017.

WANG YU-ren. Applying the PDRI in Project Risk Management. Austin, 2002.Tese (Doutorado) Faculty of the Graduate School of The University of Texas at Austin. 